\title{
Prueba psicométrica de perfiles vocacionales de Administración de Tecnología de Información, Agronegocios y Arquitectura: construcción y validación
}

Alfaro-Barquero, Alejandra; Chinchilla-Brenes, Sonia

Prueba psicométrica de perfiles vocacionales de Administración de Tecnología de Información, Agronegocios y Arquitectura: construcción y validación

Revista Educación, vol. 45, núm. 2, 2021

Universidad de Costa Rica, Costa Rica

Disponible en: https://www.redalyc.org/articulo.oa?id=44066178034

DOI: https://doi.org/10.15517/revedu.v45i1.43570

\section{(c) (1) $\Theta \Theta$}

Esta obra está bajo una Licencia Creative Commons Atribución-NoComercial-SinDerivar 3.0 Internacional. 


\section{Prueba psicométrica de perfiles vocacionales de Administración de Tecnología de Información, Agronegocios y Arquitectura: construcción y validación}

Vocational Profile Psychometric Testing for Information Technology Management, Agribusiness and Architecture Career Paths: Construction and Validation

Alejandra Alfaro-Barquero

Instituto Tecnológico de Costa Rica, Costa Rica

alealfaro@itcr.ac.cr

DOI: https://doi.org/10.15517/revedu.v45i1.43570

Redalyc: https://www.redalyc.org/articulo.oa?

iD https://orcid.org/0000-0002-8901-7129

Sonia Chinchilla-Brenes

Instituto Tecnológico de Costa Rica, Costa Rica

schicnchilla@itcr.ac.cr

(D) https://orcid.org/0000-0002-1100-2568

Recepción: 07 Septiembre 2020

Aprobación: 10 Diciembre 2020

\section{Resumen:}

La presente investigación, de corte instrumental, pretende definir el perfil vocacional de las carreras de Arquitectura (AU), Administración de Tecnología de Información (ATI) e Ingeniería en Agronegocios (AA) del Instituto Tecnológico de Costa Rica (ITCR) y construir un instrumento psicométrico para evaluar las preferencias por dichas carreras. Participaron 449 estudiantes con una media de edad de 21.29 años, $44 \%$ mujeres y $56 \%$ varones, y 22 docentes. Esta investigación da continuidad a los estudios realizados por Alfaro-Barquero y Chinchilla-Brenes $(2016,2017,2019,2020)$. Los perfiles vocacionales se elaboraron a partir de las descripciones brindadas por estudiantes y docentes; posteriormente fueron validados con grupos de personas expertas. Con la información obtenida, se construyeron los ítems de los intereses y tareas propias de las áreas de ATI, AU y AA, que se aplicaron a un grupo de estudiantes de cada carrera junto con los instrumentos de Evaluación de Satisfacción Vocacional y la Escala de habilidades de Alfaro-Barquero y Chinchilla-Brenes (2016, 2017, 2019, 2020). Las escalas utilizadas mostraron adecuados indicadores psicométricos, a excepción de la escala de intereses. Se evidenciaron diferencias significativas según sexo y carrera. También se encontró asociación entre la satisfacción vocacional y las habilidades, intereses y tareas vocacionales.

Palabras ClaVE: Intereses vocacionales, Prueba psicométrica, Tareas ocupacionales, Habilidades, Ingeniería, Arquitectura.

\section{Abstract:}

The objective of this instrumental study was to define the vocational profile of three career paths at the Costa Rican Institute of Technology (ITCR): Architecture, Information Technology Management and Agribusiness Engineering, and develop a psychometric vocational test to assess preferences for each area of study. The sample consisted of 22 teachers and 449 students with an age average of 21.29 years; $44 \%$ were female and $56 \%$ male. This study provides continuity to previous studies by AlfaroBarquero and Chinchilla-Brenes $(2016,2017,2019,2020)$. Professional profiles for each career path were obtained from students and teachers and then validated by a group of experts. The information gathered set the foundation for the interests and specific tasks related to Architecture, Information Technology Management and Agribusiness Engineering which were used as input to develop a survey that was answered by the study participants. The group also completed the Vocational Satisfaction Evaluation Test and the Skills Scale, by Alfaro-Barquero and Chinchilla-Brenes (2016, 2017, 2019, 2020). All the scales that were used appropriate psychometric indicators, except for the interest scale. Significant differences according to sex and career were evident. An association was also found among vocational satisfaction and vocational skills, interests, and tasks.

KEYWORDs: Vocational Interests, Psychometric Testing, Occupational Tasks, Skills, Engineering, Architecture. 


\section{INTRODUCCIÓN}

El proceso de toma de decisiones vocacionales es de gran trascendencia en el proyecto de vida de las personas, ya que la profesión que se elija tendrá efectos en las demás áreas de la vida y en la autorrealización.

No obstante, estas decisiones deben tomarse a edades muy tempranas y, en muchos casos, sin un adecuado proceso vocacional (Barrera, 2016). A esto se suman algunas variables que dificultan la elección, como la clase social (Estrada, 2011; Sevilla, Puerta y Dávila, 2010), la falta de información de carreras no tradicionales (Restrepo, Carvajal y Roldán 2016), la saturación de carreras en el mercado laboral y las pruebas de admisión (Carrasco, Zúñiga y Espinoza, 2014).

Estas carencias persisten en los estudios superiores, pues muchas personas jóvenes que ingresan a la universidad muestran elevados niveles de inseguridad, y pocas coincidencias entre sus intereses y la carrera elegida (Vargas y Huamán, 2010).

Esta realidad no es ajena al ITCR, según el estudio de Hernández-Jiménez, Moreira-Mora, Solís-Salazar y Fernández-Martín (2020), un $46.6 \%$ de quienes desertaron de la universidad no había ingresado a la carrera deseada. Esto se refleja también en las 606 solicitudes de cambio de carrera para 2019 mediante promedio ponderado (Carlos Araya, comunicación personal, 12 de mayo 2020) y en las 239 a través de examen de admisión (Geovanni Rojas, comunicación personal, 29 de mayo 2020), lo que representa un $8.64 \%$ de la población estudiantil activa en 2019.

Lo anterior sugiere la necesidad de fortalecer los procesos de asesoría vocacional universitaria. En este sentido, la presente investigación pretende desarrollar perfiles y pruebas vocacionales, que apoyen el proceso de toma de decisiones vocacionales en el área científico-tecnológica.

Aunque existe diversidad de instrumentos de apoyo en la elección vocacional, como la Escala de Intereses Vocacionales de Kuder, (Woyno y Oñoro, 2005), el Self-Directed Search o SDS (Holland, 1994), el Cuestionario de Áreas de Intereses Básicos Académico Profesionales (Hernández, 2001) y el Test Explora (Martínez, 2016), dichas pruebas no son específicas para el área científico-tecnológica.

A partir de esta realidad, el presente estudio da continuidad a las investigaciones realizadas por AlfaroBarquero y Chinchilla-Brenes (2016, 2017, 2019 y 2020), quienes desarrollaron los perfiles vocacionales (habilidades, tareas e intereses) para 15 carreras del ITCR. Se tiene como objetivo construir los perfiles vocacionales de las áreas de ATI, AU y AA, y, posteriormente, crear las escalas psicométricas de intereses y tareas para dichas carreras, con miras a la ampliación del instrumento Tareas, intereses y habilidades en el área de ingeniería en el ITCR, desarrollado por Alfaro-Barquero y Chinchilla-Brenes (2016, 2017, 2019, 2020).

En las siguientes páginas se retomarán aspectos conceptuales de base para el estudio y se indicarán aspectos metodológicos, así como la presentación de los resultados que respaldan las conclusiones.

\section{ANTECEDENTES}

En el proceso de toma de decisiones vocacionales, la persona considera sus características individuales (intereses, sexo, preferencias y habilidades), la oferta académica y demanda laboral, para tomar decisiones en pro de un adecuado ajuste a la carrera elegida y mayor satisfacción en el ejercicio profesional futuro.

Buzzetta, Hayden y Ledwith (2017) consideran que las habilidades como análisis, síntesis, capacidad de anticipación y autoconocimiento para la toma de decisiones vocacionales, pueden ser aprendidas y son susceptibles de entrenamiento, lo que destaca la relevancia de la asesoría vocacional en este proceso.

El análisis de los factores socioeconómicos, familiares y los relacionados con las carreras, así como salud, discapacidades, características físicas, creencias, personalidad, valores, conocimiento sobre el medio laboral, medios de comunicación, instituciones educativas, condiciones socioeconómicas y zona geográfica (McMahon y Patton, 2018) influyen en la elección vocacional al igual que la asesoría profesional, la 
autoconfianza y las competencias ocupacionales (Álvarez, Aguilar, Fernández y Sicilia, 2014; Carrasco et al., 2014; Cepero, 2009; Espíndola, 2015; Hernández, 2001; Herrera y Burgoa, 2012).

Estos factores también permiten mantener o ajustar las expectativas y metas ocupacionales e influyen en la persistencia y logros académicos, pues inciden en la valía personal y los deseos de superación en el área académica elegida (Carrasco et al, 2014; Gallego, Correa y Barragán, 2012; Lent, Hackett y Brown, 2004; Rodríguez, Inda y Peña, 2015).

En lo que se refiere al ingreso y permanencia a la educación universitaria costarricense, según datos del Programa Estado de la Nación (PEN, 2019a), desde 2009 no mejora el porcentaje de estudiantes entre 25 y 34 años que cuentan con educación superior (28 \% en contraposición al $44.5 \%$ de los países de la Organización para la Cooperación y el Desarrollo Económico) y el número de títulos que se otorga por año ha disminuido de 50000 diplomas en 2014 a 46629 en 2017, excepto en las áreas de Artes, Letras, Ingenierías y Ciencias Básicas.

Lo anterior parece estar asociado a factores demográficos y socioeconómicos, que obligan a las personas a una más temprana inserción laboral y señalan la necesidad de que las universidades estatales mejoren su gestión institucional (PEN, 2019a) y las condiciones de asesoría vocacional, acceso y permanencia.

Otro factor relevante en la continuidad de estudios universitarios es la coincidencia entre las condiciones intrapersonales (autoeficacia, expectativas de resultados y metas) y las condiciones del sistema educativo, ya que "la percepción que tiene una persona sobre su capacidad para tener éxito en los estudios de ingeniería determina el interés hacia dichos estudios y la persistencia” (Rodríguez et al, 2015, p. 270), así como la forma positiva o negativa en que valora los resultados que obtendrá en su carrera.

Hallazgos similares reportaron Schoenfeld, Segal y Borgia (2017), quienes encontraron un efecto directo de la percepción de autoeficacia sobre las expectativas de logro de resultados, lo que influye en una mayor probabilidad de graduarse, aún en condiciones socioeconómicas adversas, y de trascender estas restricciones culturales (McMahon y Patton, 2018; Richardson, 2017).

Dentro de los factores asociados con la elección vocacional, Cepero (2009) señala de mayor relevancia las preferencias e intereses vocacionales, las habilidades y el sexo; en esta línea se han encontrado diferencias en las habilidades según sexo. Echavarri, Godoy y Olaz (2007) encontraron diferencias a favor de los varones en las medias de razonamiento verbal, cálculo y razonamiento abstracto, y a favor de las mujeres en las pruebas de ortografía, lenguaje y en el rendimiento académico. Además, se evidenció que los varones se perciben con mayores habilidades físico-mecánicas; mientras que las mujeres sobresalen en sociabilidad y autorregulacióndisciplina (Alfaro-Barquero y Chinchilla-Brenes, 2016, 2017).

Las diferentes disciplinas del área científico-tecnológica que ofrece el ITCR muestran ciertas habilidades generales comunes: física, lógico-matemática, química, biología, viso-espacial, liderazgo y autorregulacióndisciplina (Alfaro-Barquero y Chinchilla-Brenes, 2016, 2017, 2019, 2020); sin embargo, los requerimientos en los niveles de dichas habilidades pueden variar entre carreras según su grado de especialización, por ejemplo, en Diseño Industrial se requiere mayor habilidad viso-espacial que lo que se necesita en otras áreas (Alfaro-Barquero y Chinchilla-Brenes, 2016).

La correspondencia entre las destrezas de la persona y los requerimientos de la carrera elegida, en términos de habilidades, incidirán en la satisfacción con la elección vocacional realizada y en sentimientos de autoestima y seguridad (Hulin, 2002, citado por Patton y McMahon, 2017).

Por otra parte, las preferencias vocacionales también inciden en la toma de decisiones y se ven afectadas por estereotipos sexistas, lo que reduce las oportunidades de acceso a carreras de corte científico-tecnológico y la posterior inserción laboral para las mujeres (Rosado, 2012). Por ejemplo, "En carreras como Física, Computación, Ingeniería Mecánica, Ingeniería Electrónica e Ingeniería Eléctrica, más del $80 \%$ de la matrícula corresponde a hombres" (PEN, 2019b, p. 48). Esta disparidad se evidenciará también en la graduación de las universidades. Según datos de 2017 (PEN, 2019a), la graduación general en universidades estatales fue del $62 \%$, el $52 \%$ de la graduación correspondió a mujeres y solo el $30 \%$ de los títulos obtenidos 
por ellas pertenece al área de ingeniería. Este dato es congruente con el porcentaje de mujeres matriculadas en el ITCR: $34 \%$ en 2019 y $33.74 \%$ en 2020 (Xiomara Salas, comunicación personal, 8 de mayo 2020).

En concordancia con lo anterior, Adelfang, 2016; Donoso-Vázquez, 2012; Rodríguez, 2016; Rosado, 2012 , indican que la representación de mujeres en el campo de la ciencia y la tecnología en el mercado laboral ronda el $30 \%$, lo que se refleja también en menores oportunidades de trabajo. Para 2019, la tasa de participación laboral en Costa Rica fue del 50.4 \% para las mujeres en edad de trabajar, mientras que la participación masculina fue del $73.1 \%$. El caso del desempleo fue de $15.4 \%$ para las mujeres, casi el doble que el porcentaje masculino, que se situó en 8.7 \%, según el (Instituto Nacional de Estadística y Censos INEC, 2019).

\section{MARCo TEÓRICo}

La adecuada elección vocacional requiere un proceso de toma de decisiones autónoma que parte de la percepción que la persona tiene de sí misma y el futuro desempeño de un rol social-ocupacional (Cepero, 2009; Müller, 1992). Debe considerar el análisis de la oferta académica y la demanda laboral; así como la adecuada correspondencia entre la carrera elegida y los rasgos individuales, tales como intereses, sexo, preferencias y habilidades (Bohoslavsky, 1984).

Los intereses, según Bohoslavsky (1984), predisponen la tendencia de la persona a sentirse motivada por un área de la realidad de un modo discriminativo en relación con otras y refieren al componente afectivo, lo que despierta curiosidad y deseo hacia el objeto de interés (Montero, 2005, Rodríguez, 2002) y se definen como "incentivadores del aprendizaje (...) aquello que agrada, preocupa o mueve a la curiosidad" (Rodríguez, 2002, p. 94). Se evidencian a través de las acciones, los valores y pautas de comportamiento e involucran tres dimensiones: cognitiva, afectiva y conductual (Montero, 2005). Estos intereses orientan a la persona a buscar ocupaciones según tareas vocacionales, así se perfilan por una u otra ocupación (Buzzetta et al., 2017; McMahon y Watson, 2015).

Las habilidades, por su parte, se conceptualizan como el nivel de capacidad para el aprendizaje o desempeño de una tarea, y se miden de forma indirecta a través de la ejecución (Super y Crites, citado por Montero, 2005). Alfaro-Barquero y Chinchilla-Brenes (2016, 2017, 2019 y 2020) identificaron 7 habilidades relacionadas con el área de ingeniería: matemática, físico-mecánica, biología, química, liderazgo, viso-espacial y autorregulación-disciplina.

La elección vocacional, así como los intereses y habilidades, son expresiones de la personalidad del sujeto y, por ende, las personas que se desempeñan en un campo profesional muestran personalidades similares. Desde el enfoque de Holland (citado por Cepero, 2009), la satisfacción, la estabilidad y el logro asociado a la elección vocacional serán el resultado de la congruencia entre la personalidad y el campo de trabajo en el que se desempeñará.

Por lo tanto, la afinidad entre las características de la profesión elegida y las metas, intereses o capacidades de la persona se reflejarán en el grado de satisfacción vocacional (Benítez, 2010). A mayor afinidad, mayor satisfacción.

Las tareas ocupacionales son las principales actividades, acciones o roles que desempeña en la práctica cotidiana el profesional en su especialidad y cobran relevancia pues le indican, a la persona que está eligiendo carrera, qué hará en el futuro para optar por una u otra carrera en congruencia con sus preferencias.

Las preferencias vocacionales son la manifestación explícita de las personas sobre su grado de predilección por una o varias actividades o profesiones (Cepero, 2009).

Si bien los seres humanos son capaces de desempeñarse en distintas ocupaciones, las preferencias vocacionales los orientan hacia áreas específicas y a "la selección de un determinado campo académicoprofesional compuesto de un conjunto de actividades lúdico-laborales (...) para el cual existen una 
serie de habilidades, aptitudes, conocimientos previos y motivos que llevan a una elección de profesión futura" (Cepero, 2009, p.24).

Por tanto, las preferencias vocacionales son el resultado de un proceso vital que se relaciona con diferentes variables contextuales, personales, familiares, socioeconómicas y educativas, pero no necesariamente responden a un adecuado nivel de autoconocimiento (valores, habilidades, intereses, características de personalidad, aptitudes, entre otras), ni se corresponden con información pertinente de los perfiles vocacionales de las carreras que ofrece el mercado ni de las condiciones de demanda y desempeño laboral (tareas ocupacionales).

Desde esta perspectiva, se considera que las preferencias vocacionales deben ser sometidas a análisis a través de un proceso de asesoría para la toma de decisiones, de tal manera que las preferencias reflejen una adecuada correspondencia entre las características de la persona y los requerimientos de la carrera, lo que más adelante se materializará en el trabajo, pues este es fuente de identidad, autonomía y espacio para el desarrollo personal (Hulin, 2002, citado por Patton y McMahon, 2017).

Aunque se asume que el grupo estudiantil de ingeniería comparte, por ejemplo, intereses y habilidades investigativas para las ciencias básicas y habilidades de razonamiento lógico-matemático (Alfaro-Barquero y Chinchilla-Brenes, 2017, 2016), las tipologías de personalidad planteadas por Holland (1994): investigativa, artística, social, convencional, emprendedora y realista, son demasiado amplias como para permitir la adecuada discriminación entre carreras afines en ingeniería (Alfaro-Barquero y Chinchilla-Brenes, 2016, 2017), por lo que se hace necesario contar con perfiles más específicos y adecuados a las características de cada carrera.

Para los fines de este estudio, el perfil vocacional de las carreras parte de la conceptualización construida por estudiantes y docentes de cada disciplina, e incluyen las siguientes áreas: a. definición de la carrera, sus objetivos y áreas de especialidad, b. la descripción de las tareas ocupacionales propias del ejercicio profesional de cada disciplina, c. la definición de temáticas de interés afines a la carrera, d. las habilidades implicadas en el proceso de formación académica y e. los contenidos de ciencias básicas relevantes en cada disciplina (AlfaroBarquero y Chinchilla-Brenes, 2016, 2017, 2019, 2020).

\section{Metodología}

La presente investigación retomó los procedimientos metodológicos desarrollados por Alfaro-Barquero y Chinchilla-Brenes $(2016,2017,2019,2020)$, basados en un enfoque mixto que combinó técnicas cualitativas y cuantitativas para definir los perfiles vocacionales de carreras de ingeniería y construir un instrumento psicométrico para evaluar las preferencias por dichas carreras. Para la definición de los perfiles vocacionales se implementaron estrategias cualitativas y para el diseño y evaluación de las escalas de tareas e intereses estrategias cuantitativas, ya que se desarrolló como investigación de tipo instrumental al incluir la construcción de un instrumento psicométrico.

\section{Participantes}

La población estuvo constituida por 22 docentes y 449 estudiantes con una media de edad de 21.29 años, $44 \%$ mujeres y 56 \% varones, 137 estudiantes de Administración de Tecnología de Información (ATI), 177 de Arquitectura (AU) y 135 de Ingeniería en Agronegocios (AA). La muestra representó un 68 \% de la población total activa de las tres carreras con 2 o más años de permanencia. Se incluyeron en el estudio tres submuestras según cada fase. 
En la fase 1: construcción del perfil vocacional según criterio de personas expertas, se trabajó con dos sub grupos; el primero constituido por 116 estudiantes ( 51 mujeres y 65 hombres), y el segundo con 20 estudiantes (13 mujeres y 7 hombres) y 22 docentes expertos y expertas ( 6 mujeres y 16 hombres).

Los criterios de selección para personas expertas fueron, en el caso de estudiantes: ser estudiante de nivel avanzado, promedio ponderado superior al percentil 75 y estar satisfecho o satisfecha con su elección vocacional. Para el personal docente: dos años de experiencia docente, haber obtenido una calificación del desempeño igual o superior a 80 y preferible con experiencia laboral en su área de especialidad.

En la fase 2: elaboración y aplicación del instrumento vocacional en versión piloto, se aplicó el instrumento a 105 estudiantes, con edad promedio de 20.39 años $(D E=1.95)$, $49.52 \%$ mujeres y $50.48 \%$ varones.

En la fase 3: aplicación del instrumento vocacional completaron el instrumento 208 estudiantes en grupos seleccionados al azar. La edad promedio fue de 22.2 años $(D E=2.1), 39.23 \%$ fueron mujeres, 60.77 $\%$ varones.

\section{Instrumentos}

Se utilizaron 3 instrumentos: a. el instrumento de recolección de información sobre tareas, intereses y habilidades según carrera (Alfaro-Barquero y Chinchilla-Brenes, 2016), b. la escala de evaluación de satisfacción vocacional (Alfaro-Barquero y Chinchilla-Brenes, 2016) y c. la escala de habilidades del instrumento de Tareas, intereses y habilidades en el área de ingeniería en el ITCR (Alfaro-Barquero y Chinchilla-Brenes, 2016, 2017 y 2019, 2020), a la cual se agregaron las escalas de tareas e intereses desarrolladas para las carreras de AA, ATI y AU.

El instrumento de recolección de información sobre tareas, intereses y habilidades (Alfaro-Barquero y Chinchilla-Brenes, 2016) se aplica de manera individual con preguntas abiertas que indagan la definición de la carrera y las áreas profesionales, la descripción de las tareas ocupacionales según áreas de trabajo, habilidades, intereses y contenidos de ciencias básicas relevantes para la carrera.

Por su parte, la evaluación de satisfacción vocacional (Alfaro-Barquero y Chinchilla-Brenes, 2016) es una escala de aplicación individual tipo Likert, que contiene ocho afirmaciones que miden el nivel de satisfacción con la profesión elegida y la visualización a futuro del rol profesional en las que las personas deben indicar su grado de acuerdo con cada ítem en una escala de respuesta de 1 a 5 . Dicha escala reportó un coeficiente de confiabilidad alfa de Cronbach de 0.74 (Alfaro-Barquero y Chinchilla Brenes 2016, 2017).

Finalmente, la escala de habilidades del instrumento de tareas, intereses y habilidades en el área de ingeniería en el ITCR se aplica individualmente e incluye 7 variables: lógico- matemática, física, liderazgo, autorregulación-disciplina, viso-espacial, biológica y química. Dicha escala reporta valores de Alfa de Cronbach superiores a 0.85 (Alfaro-Barquero y Chinchilla-Brenes, 2016, 2017, 2019, 2020). Cabe aclarar que esta investigación da continuidad a los estudios de Alfaro-Barquero y Chinchilla-Brenes (2016, 2017, 2019, 2020) que incluyen las escalas de habilidades, intereses y tareas para 12 de las 21 carreras que actualmente ofrece el ITCR, con el objetivo de construir las escalas de intereses y tareas correspondiente a las carreras ATI, AU, AA. Con respecto a las habilidades no se consideró necesaria la construcción de un nuevo instrumento, dado que el de Alfaro-Barquero y Chinchilla-Brenes (2016, 2017, 2019, 2020) ha demostrado adecuadas evidencias de confiabilidad y validez para la medición de habilidades de doce carreras del ITCR. Además, en la fase cualitativa de construcción del perfil vocacional no se vislumbró la necesidad de incorporar nuevas habilidades.

Se construyeron las escalas de tareas (61 ítems) e intereses (43 ítems) para ATI, AU y AA. En todos los casos, los valores del coeficiente de confiabilidad Alfa de Cronbach fueron superiores a 0.95, como se muestra en la sección de resultados. El instrumento, en su versión final con las tres escalas (habilidades, tareas 
e intereses), contó de 130 ítems con afirmaciones tipo Likert, con opciones de respuesta numérica de 1 a 6 para indicar el grado de acuerdo con cada ítem, donde 1 representa el menor grado de acuerdo y 6 el mayor.

El perfil vocacional incluye una lista detallada de las habilidades, intereses y tareas que corresponden a cada disciplina, de las cuales se seleccionan algunas de las más representativas para la construcción del instrumento. En la Tabla 1 se ofrece un ejemplo de cada tipo de ítem para las tres escalas utilizadas para la carrera de ATI:

TABLA 1

Ejemplo de ítems de las escalas.

\begin{tabular}{|l|l|}
\hline Área & Ejemplo de ítem en el instrumento psicométrico \\
\hline Tarea & $\begin{array}{l}\text { Quisiera trabajar en la gestión del proceso de adquisiciones de nuevas } \\
\text { tecnologías de información (cotizar, tramitar el proceso de compra, entre } \\
\text { otros) de acuerdo con los estándares de calidad y usabilidad. }\end{array}$ \\
\hline Interés & $\begin{array}{l}\text { Me interesa conocer sobre procesos de servicio al cliente en la gestión de } \\
\text { tecnologias de información. }\end{array}$ \\
\hline Habilidad & $\begin{array}{l}\text { Tengo habilidad para hacer deducciones a partir de un conjunto de datos } \\
\text { numéricos. }\end{array}$ \\
\hline
\end{tabular}

Fuente: Elaboración propia.

Los resultados de cada subescala (tareas, habilidades, intereses) del instrumento se interpretan con base en la definición de percentiles en cinco categorías, según el comportamiento muestral: 1 . Muy bajo: menor o igual al percentil 10; 2. Bajo: mayor al percentil $10 \mathrm{y}$ menor o igual al percentil 25; 3. Moderado: mayor al percentil 25 y menor al percentil 75; 4 . Alto: Igual o mayor al percentil 75 y menor al percentil 90; 5 . Muy alto: igual o mayor al percentil 90.

\section{Procedimientos}

La recolección de información se realizó en tres momentos o fases.

Fase de construcción del perfil vocacional.

En esta etapa se aplicó el instrumento de recolección de información sobre tareas, intereses y habilidades según carrera (Alfaro-Barquero y Chinchilla-Brenes, 2016). La información obtenida fue integrada y organizada por las investigadoras en la versión inicial del perfil vocacional de cada carrera. Posterior a la fase de sistematización, se realizaron grupos focales con estudiantes expertos y expertas; se concluyó con el grupo de docentes expertos y expertas que validaron el perfil vocacional.

\section{Fase de aplicación de la prueba piloto}

En esta fase se aplicó, en modo pilotaje, la escala de habilidades tomada de la prueba tareas, intereses y habilidades en el área de ingeniería en el ITCR, compuesta por la escala de habilidades (Alfaro-Barquero y Chinchilla-Brenes, 2016, 2017, 2019 y 2020) y las escalas de tareas e intereses para las carreras de la muestra, construidas en el estudio a partir de los perfiles vocacionales elaborados en la fase anterior. Además, se aplicó la escala de evaluación de satisfacción vocacional (Alfaro-Barquero y Chinchilla-Brenes, 2016).

La información obtenida se registró en una base de datos SPSS para el análisis de sus características psicométricas. En el proceso se excluyeron los ítems que no cumplieron con los estándares psicométricos requeridos y se incorporaron mejoras de redacción. La versión revisada quedó conformada por 177 ítems.

Fase de aplicación final del instrumento.

Una vez incorporadas las correcciones del instrumento, se aplicó nuevamente la versión mejorada de la escala de habilidades tomada del instrumento de tareas, intereses y habilidades en el área de ingeniería en el ITCR (Alfaro-Barquero y Chinchilla-Brenes, 2016, 2017, 2019 y 2020), las escalas de tareas e intereses 
para las carreras de la muestra, junto con la escala Evaluación de satisfacción vocacional (Alfaro-Barquero y Chinchilla-Brenes, 2016, 2017, 2019 y 2020).

\section{Análisis de datos}

En la etapa uno del estudio, las respuestas de los grupos participantes fueron digitadas textualmente y se integraron posteriormente en un mismo archivo formato Word con los siguientes apartados: 1. definición de la carrera, 2. áreas profesionales, 3. tareas ocupacionales por área, 4. áreas profesionales afines, 5. intereses, 6. habilidades y 7. contenidos de ciencias básicas.

Seguidamente se realizaron seis reuniones de grupo focal, de aproximadamente tres horas cada una, con el estudiantado experto de cada carrera; se utilizó como base para el trabajo el documento anteriormente indicado. En las discusiones se buscó consenso en torno al perfil vocacional, en procura de mejorar la estructura y coherencia de la información; se eliminaron contenidos irrelevantes, inadecuados o imprecisos, con el fin de lograr una redacción pertinente para la población no experta y estudiantes de nivel de secundaria. El producto del análisis con estudiantes expertas y expertos fue mejorado y validado con el grupo de docentes en cinco reuniones con una duración aproximada de 3 horas cada una.

En la segunda fase, todas las escalas aplicadas se evaluaron con procedimientos psicométricos: discriminación por ítem, confiabilidad de las escalas y análisis factoriales exploratorios; así como la revisión y mejora de redacción de los ítems. Los análisis factoriales se utilizaron con el propósito de resumir un conjunto de ítems (o indicadores observables) en un menor número de variables teóricas o latentes denominados factores, y se consideran procedimientos idóneos para obtener evidencias de validez de constructo (MartínezArias, 1996).

Para el análisis de los resultados de la aplicación final de las escalas, además de los procedimientos psicométricos anteriormente empleados, se realizaron Análisis Factoriales Confirmatorios (con el programa EQS 6.1 y el método de estimación de Máxima Verosimilitud). Como su nombre lo indica, tales procedimientos confirman "los factores hipotetizados en un modelo propuesto a priori" (Cea, 2002, p.430), a partir del análisis de datos empíricos; se supuso que los ítems con alta correlación entre sí, compartirían una misma estructura latente.

Para la valoración de la bondad de ajuste de los modelos hipotéticos propuestos, se utilizaron los siguientes criterios: RMSEA (la raíz cuadrada del error de estimación), con un valor esperado inferior o equivalente a 0.05; aunque se considera aceptable un valor inferior a 0.08; el CFI (índice de ajuste comparativo), con valores iguales o superiores a 0.09 , y la Chi cuadrada $\left(X^{2}\right)$, que se espera sea pequeña y con probabilidad superior a 0.05 . Sin embargo, es factible obtener valores menores a 0.05 cuando algunas variables no muestren distribución normal (Cea, 2002).

Para mejorar el ajuste del modelo, se aplicó la prueba múltiple de la Lagrange (LM test), disponible en el software EQS, que usualmente se emplea "con el fin de evaluar errores de especificación del modelo e identificar los parámetros que contribuirán a un descenso significativo del chi-cuadrado” (Carvalho, Corbella y Matos, 2011, p.95).

Finalmente, se realizó el análisis de varianza con apoyo del programa SPSS según carrera, sexo y nivel de satisfacción vocacional. En el caso de las carreras, el interés fue comprobar la existencia de diferencias significativas entre las puntuaciones de habilidades, intereses y tareas por disciplina. Por su parte, la variable de sexo se incluye en el análisis de varianza por su relevancia en las preferencias vocacionales, de acuerdo con lo anotado en los antecedentes. También se realizó el análisis de varianza según satisfacción vocacional, para identificar si existe mayor nivel de satisfacción en estudiantes con altas puntuaciones en habilidades, tareas e intereses afines a la carrera. 
Alejandra Alfaro-Barquero, et al. Prueba psicométrica de perfiles vocacionales de Administración d...

\section{Resultados}

Los resultados del perfil vocacional se redactaron ampliamente para cada carrera en un archivo de texto que se entregó a las direcciones de las carreras participantes y se resumen a modo de ejemplo en la Tabla 2. 
Revista Educación, 2021, vol. 45, NúM. 2, Julio-Diciembre, ISSN: 0379-7082 2215-2644.

TABLA 2

Síntesis del perfil vocacional.

\begin{tabular}{|c|c|c|c|c|}
\hline \multicolumn{5}{|c|}{ Administración de Tecnología de Información } \\
\hline Descripción ATI & Áreas & Tareas ocupacionales & Áreas afines & Intereses/habilidades \\
\hline $\begin{array}{l}\text { Disciplina que busca mejorar el } \\
\text { rendimiento de la empresa y el } \\
\text { logro de sus objetivos } \\
\text { estratégicos mediante la } \\
\text { sofisticación y optimización de } \\
\text { tecnología de información (TI), } \\
\text { administración eficiente y } \\
\text { eficaz de los recursos. }\end{array}$ & $\begin{array}{l}\text { Alineación } \\
\text { estratégica de TI. } \\
\text { Integración y } \\
\text { automatización de } \\
\text { procesos. } \\
\text { Gestión de } \\
\text { sistemas y } \\
\text { servicios de TI. } \\
\text { Inteligencia de } \\
\text { negocios. }\end{array}$ & $\begin{array}{l}\text { Valorar el estado actual de la } \\
\text { organización para identificar } \\
\text { necesidades y áreas de mejora en TI. } \\
\text { Evaluar y proponer mejoras a los } \\
\text { procesos de negocios: contabilidad, } \\
\text { recursos humanos, producción, etc. } \\
\text { Cumplir y mejorar los estándares y } \\
\text { politicas de seguridad informática según } \\
\text { normativas. } \\
\text { Integrar fuentes de datos separadas para } \\
\text { ofrecer a la persona usuaria un sistema } \\
\text { de consultas de información. }\end{array}$ & $\begin{array}{l}\text { I. Computación I. } \\
\text { Electrónica I. } \\
\text { Producción Industrial } \\
\text { Administración de } \\
\text { Empresas I. } \\
\text { Industrial } \\
\text { Informática } \\
\text { Empresarial } \\
\text { Estadistica }\end{array}$ & $\begin{array}{l}\text { Intereses TI y programación } \\
\text { Liderazgo y planificación, } \\
\text { gestión y evaluación de } \\
\text { proyectos Habilidades } \\
\text { Pensamiento lógico /abstracto } \\
\text { Liderazgo Capacidad de } \\
\text { análisis y toma de decisiones. }\end{array}$ \\
\hline \multicolumn{5}{|l|}{ Ingeniería en Agronegocios } \\
\hline Descripción AA & Áreas & Tareas ocupacionales & Áreas afines & Intereses /habilidades \\
\hline $\begin{array}{l}\text { Carrera con enfoque } \\
\text { multidisciplinario orientada a } \\
\text { la Gestión de Agronegocios, } \\
\text { desde la producción primaria } \\
\text { hasta la comercialización de } \\
\text { bienes y servicios con } \\
\text { sostenibilidad, sustentabilidad } \\
\text { y la satisfacción de sus } \\
\text { consumidores. }\end{array}$ & $\begin{array}{l}\text { Gestión en } \\
\text { Agronegocios } \\
\text { Producción } \\
\text { primaria agricola } \\
\text { Producción } \\
\text { primaria pecuaria } \\
\text { Agroindustria }\end{array}$ & $\begin{array}{l}\text { Estudios de mercado y control de calidad } \\
\text { en procesos de producción, } \\
\text { comercialización distribución y } \\
\text { exportación. } \\
\text { Análisis y planificación de la producción } \\
\text { para minimizar costos y tomar decisiones } \\
\text { sobre métodos y técnicas de cultivo. } \\
\text { Manejo de técnicas de producción y } \\
\text { mejoramiento genético, reproducción y } \\
\text { producción de especies. } \\
\text { Conocer, implementar, innovar y evaluar } \\
\text { diferentes procesos de producción } \\
\text { agroindustrial y productos no } \\
\text { alimentarios. }\end{array}$ & $\begin{array}{l}\text { Administración de } \\
\text { Empresas I. } \\
\text { Producción Industrial } \\
\text { I. Agrícola I. } \\
\text { Agronomía I. Forestal } \\
\text { Zootecnia Tecnología } \\
\text { de Alimentos }\end{array}$ & $\begin{array}{l}\text { Intereses Gerencia e } \\
\text { innovación. Producción } \\
\text { ecoamigable Trabajo de } \\
\text { campo Habilidades } \\
\text { Innovación y } \\
\text { emprendedurismo Gestión de } \\
\text { proyectos Manejo de equipos } \\
\text { tecnológicos Química y } \\
\text { matemática. }\end{array}$ \\
\hline \multicolumn{5}{|l|}{ Arquitectura } \\
\hline Descripción AU & Áreas & Tareas ocupacionales & Áreas afines & Intereses/habilidades \\
\hline $\begin{array}{l}\text { Disciplina enfocada en la } \\
\text { búsqueda de soluciones a } \\
\text { necesidades a través del diseño } \\
\text { de espacios arquitectónicos, } \\
\text { residenciales, comerciales e } \\
\text { institucionales, según } \\
\text { requerimientos de las personas } \\
\text { usuarias y su contexto. } \\
\text { Participa en la } \\
\text { conceptualización, diseño y } \\
\text { supervisión de proyectos. }\end{array}$ & $\begin{array}{l}\text { Diseño urbano } \\
\text { Dirección y } \\
\text { administración de } \\
\text { obra } \\
\text { Conservación del } \\
\text { patrimonio } \\
\text { tangible }\end{array}$ & $\begin{array}{l}\text { Diseño arquitectónico según las } \\
\text { necesidades de sus usuarios: } \\
\text { organización de los espacios internos y } \\
\text { externos según funcionalidad, } \\
\text { estabilidad, estética, lote, contexto, etc. } \\
\text { Evaluación del proyecto y estimación del } \\
\text { presupuesto en equipos } \\
\text { interdisciplinarios. } \\
\text { Elaborar planes reguladores municipales } \\
\text { y estratégicos de ordenamiento territorial } \\
\text { (equipos interdisciplinarios). } \\
\text { Interpretar planos constructivos, dirigir e } \\
\text { inspeccionar obras en equipos } \\
\text { interdisciplinarios. } \\
\text { Analizar el patrimonio arquitectónico y } \\
\text { urbano y promover su conservación y } \\
\text { restauración. }\end{array}$ & $\begin{array}{l}\text { Paisajismo I. } \\
\text { Construcción I. Civil } \\
\text { Antropología Historia } \\
\text { Artes }\end{array}$ & $\begin{array}{l}\text { Intereses Dibujo y fotografía } \\
\text { Construcción y diseño Diseño } \\
\text { gráfico y artes Planificación } \\
\text { urbana Habilidades } \\
\text { Creatividad Análisis y } \\
\text { resolución de problemas } \\
\text { Razonamiento espacial } \\
\text { Comunicación gráfica } \\
\text { Habilidad plástica }\end{array}$ \\
\hline
\end{tabular}


Fuente: Elaboración propia.

Por otro lado, los resultados del análisis factorial confirmatorio de los instrumentos aplicados en la fase final del instrumento se detallan en la Tabla 3.

TABLA 3

Resultados de la evaluación de los modelos de medición con el análisis factorial confirmatorio y de otras características psicométricas de las subescalas.

\begin{tabular}{|c|c|c|c|c|c|c|c|c|}
\hline Escala & Ítems & Alfa de Cronbach & Discriminación mínima por ítem & $\mathrm{CFI}$ & RMSEA & Chi cuadrado & Coeficiente factorial mínima & Coeficiente factorial máxima \\
\hline Satisfacción vocacional & 8 & 0.93 & 0.59 & 1 & 0.04 & $23.46 p<.17$ & 0.61 & 0.88 \\
\hline Intereses Agronegocios & 10 & 0.98 & 0.71 & 0.99 & 0.07 & $65.3 p<.001$ & 0.71 & 0.97 \\
\hline Intereses Arquitectura & 10 & 0.97 & 0.77 & 0.99 & 0.07 & $62.56 \mathrm{p}=.001$ & 0.77 & 0.93 \\
\hline $\begin{array}{l}\text { Intereses Adm. } \\
\text { Tecnología de Inf. }\end{array}$ & 11 & 0.96 & 0.29 & 0.99 & 0.06 & $74.65 p=.001$ & 0.28 & 0.94 \\
\hline Tareas Agronegocios & 10 & 0.98 & 0.82 & 0.99 & 0.08 & $70.37 p<.001$ & 0.82 & 0.97 \\
\hline Tareas Arquitectura & 10 & 0.97 & 0.83 & 0.99 & 0.07 & $63.79 p<.001$ & 0.82 & 0.93 \\
\hline $\begin{array}{l}\text { Tareas Adm. } \\
\text { Tecnologia de Inf. }\end{array}$ & 11 & 0.97 & 0.71 & 0.98 & 0.07 & $80.74 p<.001$ & 0.71 & 0.90 \\
\hline $\begin{array}{l}\text { Habilidad viso-espacial } \\
\text { artística }\end{array}$ & 10 & 0.90 & 0.55 & 0.95 & 0.08 & $77.87 p<.001$ & 0.55 & 0.84 \\
\hline Habilidad física & 7 & 0.89 & 0.62 & 0.99 & 0.05 & $19.65 p=0.10$ & 0.63 & 0.83 \\
\hline Habilidad química & 9 & 0.97 & 0.81 & 0.99 & 0.08 & $51.58 \mathrm{p}=.001$ & 0.83 & 0.96 \\
\hline Habilidad biológica & 11 & 0.96 & 0.72 & 0.98 & 0.07 & $72.3 p<.001$ & 0.69 & 0.91 \\
\hline Habilidad liderazgo & 13 & 0.91 & 0.49 & 0.94 & 0.07 & $137.1 p<.001$ & 0.50 & 0.79 \\
\hline Habilidad disciplina & 9 & 0.82 & 0.45 & 0.96 & 0.06 & $43.89 p=.02$ & 0.44 & 0.68 \\
\hline
\end{tabular}

Fuente: Elaboración propia.

En este caso, todas las escalas obtuvieron coeficientes de confiabilidad iguales o superiores a 0.81 ; la discriminación de los ítems fue superior a 0.27, lo cual es aceptable. Además, en cada subescala se evidenció la unidimensionalidad de cada una, lo que confirma la medición de un único constructo por subescala.

Los indicadores de bondad de ajuste fueron aceptables en todos los casos, los valores de CFI fueron superiores a 0.93; los del RMSEA iguales o inferiores a 0.08 y los del Chi cuadrado fueron relativamente pequeños. Además, los coeficientes factoriales mostraron valores entre 0.28 y 0.97 , lo que se considera aceptable.

Posterior a la evaluación individual de cada subescala, se procedió a realizar los análisis confirmatorios para comprobar la estructura factorial de las escalas de tareas e intereses, producto de esta investigación. En este caso se utilizaron parcelas para evaluar cada subescala. Para ello, todos los ítems que componen una escala se dividen para obtener dos sub-puntuaciones, las cuales se utilizan como indicadores para medir la variable latente. Este procedimiento se utiliza comúnmente para simplificar la representación de los modelos.

Por otra parte, el modelo confirmatorio de la escala de intereses se muestra en la Figura 1. En este caso, el valor del error RMSEA fue mayor al criterio aceptable, por lo que, en términos estrictos, no se puede confirmar la estructura factorial. 


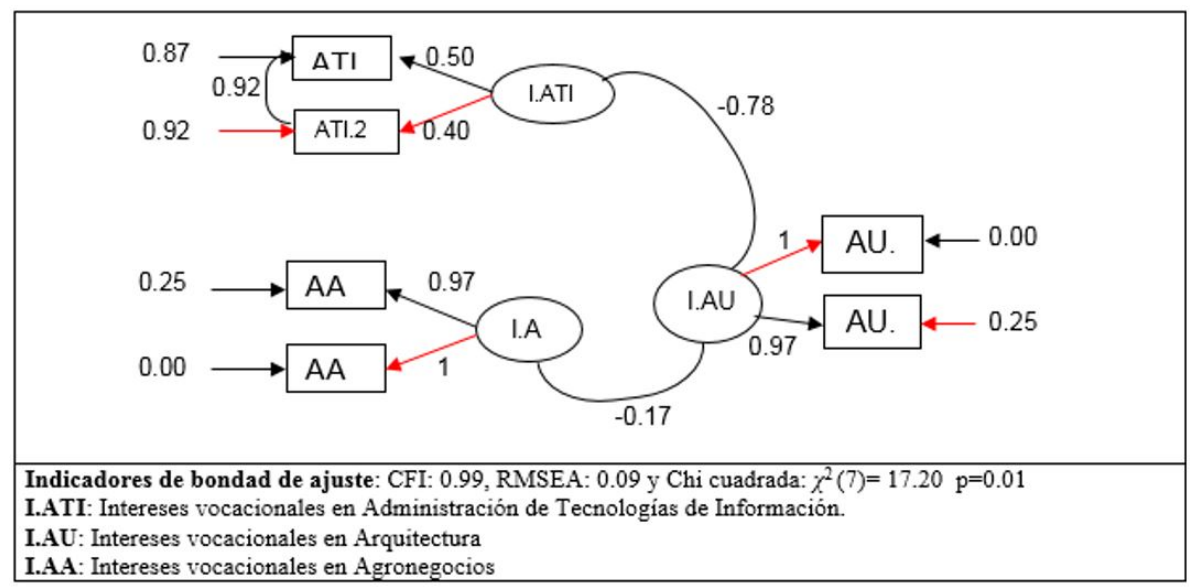

FIGURA 1.

Modelo Factorial Confirmatorio: escala de intereses vocacionales. Fuente: Elaboración propia.

Finalmente, la confirmación de la escala de tareas se presenta en la Figura 2. El modelo cumplió con todos los criterios de bondad de ajuste, así se comprobó la estructura factorial: tareas de Administración de Tecnología de Información, Tareas de Agronegocios y Tareas de Arquitectura. Una vez finalizada la evaluación de las características psicométricas de las escales, se realizaron los análisis de varianza.

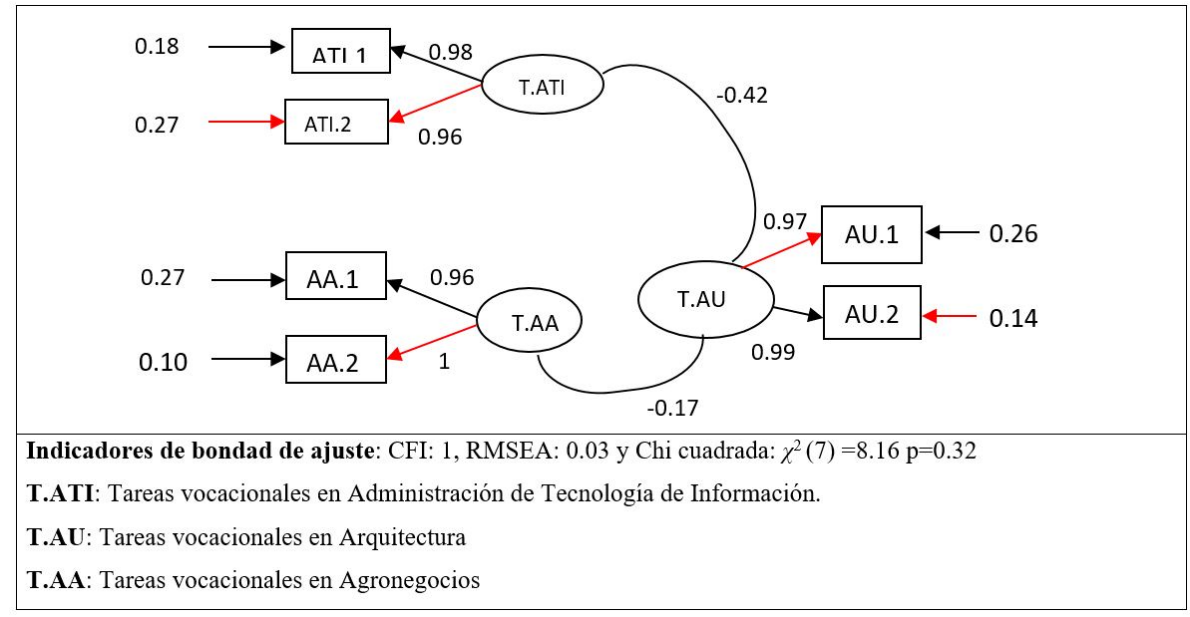

FIGURA 2 .

Modelo Factorial Confirmatorio: escala de tareas vocacionales.

Fuente: Elaboración propia.

El análisis de varianza según carrera mostró diferencias significativas en las variables habilidad viso-espacial $(F(2): 1.239, p<.001)$, habilidad matemática $(F(2): 3.767, p<.025)$, habilidad física $(F(2): 12.297, p<.001)$, habilidad biológica $(F(2): 96.659, p<.001)$, habilidad química $(F(2): 98.618, p<.001)$, liderazgo $(F(2)$ : $15.397, p<.001)$, intereses por Agronegocios $(F(2): 299.959, p<.001)$, intereses por Arquitectura $(F(2)$ : 197.341, $p<.001)$, intereses por Administración de Tecnología de Información $(F(2): 194.540, p<.001)$, tareas de Administración de Tecnología de Información $(F(2): 161.450, p<.001)$, tareas de Arquitectura $(F(2): 199.624, p<.001)$ y tareas de Agronegocios $(F(2): 275.697, p<.001)$, así como también en el promedio ponderado $(F(2): 21.986, p<.001)$.

Para analizar las diferencias entre las carreras evaluadas se implementó la prueba Tukey. Los resultados evidenciaron que el grupo de estudiantes de la carrera de Ingeniería en Agronegocios obtuvo promedios significativamente mayores que el grupo de estudiantes de Administración de Tecnología de Información 
y de Arquitectura en los intereses ( $p<.001)$ y tareas $(p<.001)$ de su área de especialidad (Agronegocios), en las habilidades biológicas $(p<.001)$, físicas $(p<.001)$, químicas $(p<.001)$ y reportaron mayores habilidades matemáticas $(p<.001)$ y de liderazgo que $(p<.001)$ el grupo de Arquitectura $(p<.001)$.

El grupo de estudiantes de Administración de Tecnología de Información obtuvo promedios significativamente mayores que los evidenciados en el caso de Ingeniería en Agronegocios y Arquitectura en los intereses $(p<.001)$ y tareas $(p<.001)$ por el área de Tecnología de Información; además, reflejaron mayores habilidades de liderazgo $(p=0.00)$ que el estudiantado de Arquitectura.

El grupo de estudiantes de Arquitectura mostró medias más altas en comparación con Administración de Tecnología de Información e Ingeniería en Agronegocios en la habilidad viso-espacial y en el promedio ponderado $(p<.001)$. Además, mostraron mayores habilidades físicas que el grupo de Administración de Tecnología de Información $(p=0.02)$. No se encontraron diferencias significativas según carrera en la satisfacción vocacional ni en la autorregulación-disciplina.

Por otra parte, el análisis de varianza según nivel de satisfacción vocacional mostró diferencias significativas en: tareas AA $(t(38):-4.392, p<.001)$, tareas AU $(t(36):-2.353, p=.024)$, tareas ATI $(t(45):-4.783, p<.001)$; intereses ATI $(t(45):-4.338, p<.001)$, intereses AU $(t(37):-2.364, p=.023)$, intereses AA $(t(38):-4.28, p<.001)$; habilidad de liderazgo $(t(124):-3.598, p<.001)$, habilidad de autorregulacióndisciplina $(t(124):-4.272, p<.001)$, habilidad biológica $(t(123):-2.147, p=.034)$, habilidad razonamiento matemático $(t(124):-2.868, p=.005)$, habilidad viso-espacial $(t(123):-2.06, p=.042)$. No se encontraron diferencias significativas en la habilidad física y química ni en el promedio ponderado.

Los resultados reflejaron que quienes se ubicaron en los niveles altos de satisfacción vocacional, también mostraron puntajes significativamente mayores en las escalas de habilidades, tareas e intereses que quienes con baja satisfacción.

Además, se encontraron diferencias significativas según sexo a favor de las mujeres en las medias de rendimiento académico ( $t(197): 2.236, p=.026$, M:75.36 mujeres, M:72.69 hombres), la habilidad visoespacial $(t(204): 3.715, p<.001$, M: 45.62 mujeres, M: 40.12 hombres), la habilidad de autorregulación ( $t(206): 2.25, p=.026, \mathrm{M}: 44.74$ mujeres, M: 42.72 hombres) y los intereses de Arquitectura ( $t(206): 2.149$, $p=.033$, M: 36.79 mujeres, M: 31.54 hombres). Por su parte, los hombres mostraron mayores puntajes en las medias de razonamiento matemático ( $t(206):-4.947, p<.001$, M: 29.6 mujeres, M: 36.45 hombres), habilidad física ( $t$ (206): -5.202, $p<.001$, M: 16.44 mujeres, M: 22.11 hombres), habilidad biológica $(t(205)$ : -2.213, $p=.028$, M: 30.14 mujeres, M: 34.91 hombres), habilidad química $(t(206):-2.806, p=.005, \mathrm{M}: 21.44$ mujeres, M: 26.67 hombres), intereses ATI ( $t(206):-4.217, p<.001$, M: 27.42 mujeres, M: 37.66 hombres) y tareas ATI ( $t(206:-4.508, p<.001$, M: 30.75 mujeres, M: 42.04 hombres). No se encontraron diferencias significativas según sexo en la satisfacción vocacional, liderazgo ni preferencia por intereses y tareas de Agronegocios.

\section{Conclusiones}

El estudio en el campo vocacional es muy amplio, no obstante, la experiencia en asesoría psicoeducativa y vocacional de Alfaro-Barquero y Chinchilla-Brenes $(2016,2017,2019,2020)$, en una universidad científico tecnológica, ha evidenciado que las pruebas vocacionales existentes son muy generales, como la prueba SDS de Holland (1994), que parte de 6 tipologías de personalidad para determinar las preferencias del estudiantado.

En este sentido, los estudios realizados por Alfaro-Barquero y Chinchilla-Brenes (2017) evidenciaron pobres indicadores psicométricos en el análisis de factores exploratorios (KMO 0.38 y 0.28) en la prueba SDS de Holland (1994), ya que únicamente encontraron la presencia de tres factores: realista, convencional, emprendedor; no se encontró asociación entre las tipologías de Holland y la carrera de Computación.

Con este panorama se hace evidente la necesidad de contar con pruebas más especializadas, sobre todo en el caso de universidades con predominancia científico-tecnológica. Es así como este estudio y otros previos 
(Alfaro-Barquero y Chinchilla- Brenes, 2016, 2017, 2019, 2020) parten de una conceptualización general de tareas, habilidades e intereses y de una perspectiva psicológica vocacional, en la cual las características personales son relevantes en la elección de carrera y, consecuentemente, la satisfacción y éxito en el desempeño profesional estarán condicionados por este supuesto.

De ese modo, el estudio tuvo como punto de partida la construcción de una conceptualización teórica para cada una de las carreras, así como la descripción de las habilidades, tareas e intereses de cada disciplina, lo cual permitió la elaboración de instrumentos psicométricos orientados a apoyar la identificación de las preferencias vocacionales de la muestra.

Los resultados del análisis psicométrico de los instrumentos utilizados (Evaluación de la satisfacción vocacional y la Escala de habilidades de Alfaro-Barquero y Chinchilla-Brenes, 2016, 2017, 2019, 2020), así como de las escalas de tareas e intereses construidas para las áreas de Agronegocios, Administración de Tecnología de Información y la de Arquitectura, mostraron adecuados indicadores psicométricos en la mayoría de los casos, y mejores que los reportados para las escalas de Holland (Alfaro-Barquero y ChinchillaBrenes, 2016, 2017, 2019, 2020).

Además, se encontraron diferencias entre estudiantes de las tres carreras en las escalas de habilidades, tareas e intereses, por lo que el instrumento puede considerarse una herramienta útil para discriminar entre las preferencias y habilidades vocacionales de estudiantes de estas tres carreras.

En la mayoría de los casos, las personas que se ubicaron en los niveles altos de satisfacción vocacional, obtuvieron mejores puntajes en las escalas de habilidades, intereses y tareas de su área, lo que respalda las afirmaciones de que la satisfacción vocacional refleja el grado de congruencia entre los intereses y características de cada persona, y los requerimientos de las carreras, es decir, como una extensión de la personalidad (Benítez, 2010 y Holland, citado por Cepero, 2009).

En este punto es importante destacar, que no se encontró asociación entre el promedio ponderado y la satisfacción vocacional. Resulta más importante para las personas la congruencia entre sus características de personalidad y la carrera elegida, independientemente de sus calificaciones.

Los resultados también ponen en evidencia las diferencias por género y cómo los hombres y mujeres tienden a mostrar preferencias y a percibirse con mayores habilidades en áreas que han tenido tradición masculina o femenina (Adelfang, 2016 y Rosado, 2012). Así, por ejemplo, se encontró que los hombres mostraron mayor predilección por la Administración de Tecnología de Información y se autoevaluaron con mayores habilidades en matemática, física y bioquímica; mientras que las mujeres mostraron mayor inclinación por el área de Arquitectura y se percibieron con mayores habilidades viso-espaciales y de autorregulación.

Sin embargo, es importante destacar que, a pesar de las discrepancias manifestadas en las preferencias vocacionales según sexo, no se encontraron diferencias significativas en los niveles de satisfacción vocacional, independientemente de la carrera elegida; en el caso de las mujeres, ellas mostraron un rendimiento académico superior a los varones. Lo anterior puede llevar a plantear la hipótesis de que el sesgo en las preferencias vocacionales responde más a un estereotipo, pues los resultados de este estudio, así como los de Alfaro-Barquero y Chinchilla-Brenes $(2016,2017,2019,2020)$ evidenciaron niveles de éxito iguales o superiores para las mujeres que estudian carreras científico-tecnológicas.

Finalmente, si bien se respalda el planteamiento de Holland que afirma que la elección de carrera responde a características de personalidad, Alfaro-Barquero y Chinchilla-Brenes (2016, 2017, 2019 y 2020) concluyeron que las tipologías planteadas por el autor son insuficientes para atender las necesidades de estudiantes que se orientan por una misma área de saber y, en particular, por el campo de la ingeniería. En este sentido, Reardon (2017) hace alusión a la teoría de Holland como simplista, pese a reconocer que la prueba vocacional es una de las más usadas en la asesoría vocacional.

En contraposición, la propuesta desarrollada en este estudio, aunque asume la existencia de similitudes entre estudiantes de áreas afines como la ingeniería, propone la existencia de tareas e intereses específicos, 
así como requerimientos de habilidades distintas según la carrera, lo que ha permitido efectivamente discriminar entre las preferencias del estudiantado en carreras científico-tecnológicas. Por lo tanto, conocer estos contrastes redundará en la oferta de procesos más sólidos desde la psicología y la asesoría vocacional.

\section{REFERENCIAS BIBLIOGRÁFICAS}

Adelfang, J. (2016, 8 de marzo). Microanálisis macro. Los datos de hoy en menos de 200 palabras. La República, p. 20.

Alfaro-Barquero, A. y Chinchilla-Brenes, S. (diciembre, 2016). Diseño de una prueba vocacional para estudiantes de las Ingenierías en Construcción, Diseño Industrial y Producción Industrial en el Instituto Tecnológico de Costa Rica (ITCR). En $V$ Encuentro sobre didáctica de la estadística, la probabilidad y el análisis de datos. Ponencia presentada en Instituto Tecnológico de Costa Rica (ITCR), Cartago, Costa Rica. Recuperado de https://bit.l y/2QQijbG

Alfaro-Barquero, A. y Chinchilla-Brenes, S. (2017). Construcción y Validación de un instrumento de evaluación de preferencias y habilidades vocacionales para carreras científico-tecnológicas. Revista Tecnología en Marcha, 30(4), 138-149. doi: https://doi.org/10.18845/tm.v30i4.3418

Alfaro-Barquero, A. y Chinchilla-Brenes, S. (2019). Diseño de un instrumento de preferencias vocacionales en Administración, Materiales y Biotecnología. Revista Costarricense de Psicología, 38(2), 99-124. doi: https://doi .org/10.22544/rcps.v38i02.01

Alfaro-Barquero A. y Chinchilla-Brenes, S. (2020). Preferencias y habilidades vocacionales de las Ingenierías Ambiental, Forestal y Seguridad Laboral e Higiene Ambiental. Revista Digital: Matemática, Educación e Internet, 20(2). doi: https://doi.org/10.18845/rdmei.v20i2.5041

Álvarez, J., Aguilar, J., Fernández, J. y Sicilia, M. (2014). El prestigio profesional y social: determinante de la decisión vocacional. Revista Española de Orientación y Psicopedagogía, 25(2), 44-50. doi: https://doi.org/10.5944/reop. vol.25.num.2.2014.13519

Barrera, M. (2016). Orientación vocacional para una acertada elección de carreras en la educación superior. Revista de investigación Académica Mucuties Universitaria, 3, 25-34. Recuperado de https://bit.ly/3gTACr5

Benítez, J. (2010). Madurez Vocacional y satisfacción académica de los estudiantes de enfermería de Luz (tesis de maestría no publicada). Universidad César Vallejo. Perú. Recuperado de https://docplayer.es/77893627-Madurez-voca cional-y-satisfaccion-academica-de-los-estudiantes-de-enfermeria-de-luz.html

Bohoslavsky, R. (1984). Orientación Vocacional: la estrategia clínica. Buenos Aires: Ediciones Nueva Visión.

Buzzetta, M., Hayden, S., y Ledwith, K. (2017). Creating hope: Assisting veterans with job search strategies using cognitive information processing theory. Journal of Employment Counseling, 54(2), 63-74. doi: https://doi.org $/ 10.1002 /$ joec. 12054

Carrasco, E., Zúñiga, C. y Espinoza, J. (2014). Elección de carrera en estudiantes de nivel socioeconómico bajo de universidades chilenas altamente selectivas. Calidad en la Educación, 40, 95-128. doi: https://doi.org/10.4067 /S0718-45652014000100004

Carvalho, H. M., Corbella, S., y Matos, P. M. (2011). Análisis Factorial Confirmatorio de la Versión Corta Portuguesa del Cuestionario del Estilo Personal del Terapeuta (EPT-C). Revista Argentina de Clínica Psicológica, 20(1), 91-102.

Cea, M. (2002). Análisis Multivariante. Teoría y práctica en la investigación social. Madrid, España: Editorial Síntesis.

Cepero, A. (2009). Las preferencias profesionales y vocacionales del alumnado de secundaria y formación profesional especifica (tesis doctoral no publicada). Universidad de Granada. España. Recuperado de https://dialnet.unirio ja.es $/$ servlet $/$ tesis? codigo $=65575$

Donoso-Vázquez, T. (octubre, 2012). Propuestas para una orientación con perspectiva de género. En I Congreso internacional e interuniversitario de Orientación Educativa y Profesional. Rol y retos de la orientación en la universidad y en la sociedad del siglo XXI. Ponencia presentada en congreso Facultad de Ciencias de la Educación, de la Universidad de Málaga, Málaga, España. Recuperado de https://bit.ly/2F43mjy 
Echavarri, M., Godoy, J. y Olaz, F. (2007). Diferencias de género en habilidades cognitivas y rendimiento académico en estudiantes universitarios. Universitas Psychologica, 6(2), 319-329. Recuperado de https://www.redalyc.org /pdf/647/64760211.pdf

Espíndola, M. (2015). Caracterización de factores: psicológico, social, económico y académico, asociados a la elección vocacional en estudiantes inscritos al curso de orientación vocacional de la UAJMS1. Revista Ventana Científica, 6(23), 23-30. Recuperado de https://bit.ly/2YWiBSU

Estrada, P. (2011). Factores que intervienen en la elección de carrera de estudiantes de bachillerato de dos modalidades educativas. En XI Congreso Nacional de Investigación Educativa. Consejo Mexicano de Investigación Educativa, México. Recuperado de https://bit.ly/3gTgiWX

Gallego, J., Correa, M. y Barragán, B. (2012). La construcción social de las profesiones: estudio de caso del Occidente Antioqueño. Uni-pluri/versidad, 12(2), 73-79. Recuperado de https://bit.ly/31SmBG1

Hernández, V. (2001). Análisis causal de los intereses profesionales en los estudiantes de secundaria (tesis doctoral no publicada). Universidad Cumplutense de Madrid. España. Recuperado de https://eprints.ucm.es/4860/1/T25 446.pdf

Herrera, E. y Burgoa, T. (2012). La importancia del perfil vocacional en la visión del estudiante universitario: caso de estudio en facultades de la Universidad Autónoma de Nueva León, UANL. DAENA: International Journal of Good Conscience, 8(2), 23-47. Recuperado de http://www.spentamexico.org/v8-n2/A2.8(2)23-47.pdf

Holland, J. (1994). Self-directed search. Forma R. (4a.ed.). Florida: Psychological Assessment Resources.

Instituto Nacional de Estadística y Censos [INEC]. (2019). Encuesta Continua de Empleo al tercer trimestre de 2019. Resultados generales. Vol. 3, Año 8. Octubre, 2019. San José, Costa Rica: Instituto Nacional de Estadística y Censos (INEC). Recuperado de https://www.inec.cr/documento/ece-2019-encuesta-continua-de-empleo-al-t ercer-trimestre-de-2019-resultados-generales

Lent, R., Hackett, G., y Brown, S. (2004). Una perspectiva social cognitiva de la transición entre la escuela y el trabajo. Revista Evaluar, 4(1). Recuperado de https://revistas.unc.edu.ar/index.php/revaluar/article/view/596

McMahon, M. y Watson, M. (Eds.). (2015). Career assessment: Qualitative approaches. Holland: Sense Publishers. Recuperado de https://silo.tips/download/qualitative-approaches

McMahon, M. y Patton, W. (2018). Systemic thinking in career development theory: contributions of the Systems Theory Framework. British Journal of Guidance \& Counselling, 46(2), 229-240. Recuperado de https://www.t andfonline.com/doi/abs/10.1080/03069885.2018.1428941

Martínez. J. (2016). Explora cuestionario para la orientación vocacional y profesional. Revista INFAD de Psicología International Journal of Developmental and Educational Psychology, 4(1), 335-343. Recuperado de http://www .infad.eu/RevistaINFAD/OJS/index.php/IJODAEP/article/view/620/558

Martínez-Arias, R. (1996). Psicometría: teoría de los test psicológicos y educativos. España: Síntesis.

Montero, P. (2005). Actualización del Inventario de Intereses Vocacionales de G. F. Kuder forma C, en estudiantes de II año de enseñanza media Cientifico-Humanista del Gran Santiago (tesis de licenciatura no publicada). Universidad de Chile. Recuperado de https://bit.ly/2YZQvGe

Hernández-Jiménez, M., Moreira-Mora, T., Solís-Salazar, M., Fernández-Martín, T. (2020). Estudio descriptivo de variables sociodemográficas y motivacionales asociadas a la deserción: la perspectiva de personas universitarias de primer ingreso. Revista Educación, 44(1), 108-127. doi: https://doi.org/10.15517/revedu.v44i1.37247

Müller, M. (1992). Orientación vocacional. Buenos Aires, Argentina: Miño y Dávila Editores.

Patton, W. y McMahon, M. (2017). The Systems Theory Framework of Career Development. En J. P. Sampson, E. Bullock-Yowell, V. C. Dozier, D. S. Osborn, y J. G. Lenz (Eds.), Integrating Theory, Research and Practice in Vocational Psychology: Current Status and Future Directions (pp. 50-61). Tallahassee, FL: Florida State University. doi: https://doi.org/10.17125/svp2016.ch4

Programa Estado de la Nación [PEN]. (2019a). Séptimo Informe Estado de la Educación Costarricense. Pavas, Costa Rica: Consejo Nacional de Rectores (CONARE), Programa Estado de la Nación (PEN). Recuperado de http s://bit.ly/2QMXaz4 
Programa Estado de la Nación [PEN]. (2019b). Resumen Séptimo Informe Estado de la Educación Costarricense. Pavas, Costa Rica: Consejo Nacional de Rectores (CONARE), Programa Estado de la Nación (PEN). Recuperado de https://bit.ly/3bnZc25

Reardon, C. (2017). Holland's Integration of Career Theory, Research and Practice. En J. P. Sampson, E. BullockYowell, V. C. Dozier, D. S. Osborn, y J. G. Lenz (Eds.). Integrating Theory, Research and Practice in Vocational Psychology: Current Status and Future Directions. (pp. 28-39). Tallahassee, FL: Florida State University. doi: h ttp://doi.org/10.17125/svp2016.ch4

Restrepo, B., Carvajal, C. y Roldán, N. (2016). Preferencias profesionales de estudiantes de educación media del Bajo Cauca, Norte y Nordeste de Antioquia, Colombia. Senderos pedagógicos, 7(7), 95-105. Recuperado de https:// ojs.tdea.edu.co/index.php/senderos/article/view/398/428

Richardson, S. (2017). Counseling for Work and Relationship: A Practice-driven Theoretical Approach. En J. P. Sampson, E. Bullock-Yowell, V. C. Dozier, D. S. Osborn, y J. G. Lenz (Eds.). Integrating Theory, Research and Practice in Vocational Psychology: Current Status and Future Directions (pp. 40-49). Tallahassee, FL: Florida State University. doi: http://doi.org/10.17125/svp2016.ch4

Rodríguez, M. (2002). Hacia una nueva orientación universitaria: modelos integrados de acción tutorial, orientación curricular y construcción del proyecto profesional. Barcelona, España: Ediciones de la Universitat de Barcelona.

Rodríguez, M., Inda, M. y Peña, J. (2015). Validación de la teoría cognitivo social de desarrollo de la carrera con una muestra de estudiantes de ingeniería. Educación XXI: Revista de la Facultad de Educación, 18(2), 257-276. doi: https://doi.org/10.5944/educxx1.14604

Rodríguez, R. (2016, 8 de marzo). Gerencia femenina, fórmula clave para éxito empresarial. La República, p. 8.

Rosado, C. (2012). Género, orientación educativa y profesional. Revista Mexicana de Orientación Educativa, 9(22), 36-41. Recuperado de http://pepsic.bvsalud.org/pdf/remo/v9n22/a06.pdf

Schoenfeld, J., Segal, G. y Borgia, D. (2017). Social cognitive career theory and the goal of becoming a certified public accountant. Accounting Education, 26(2), 109-126. doi: https://doi.org/10.1080/09639284.2016.1274909

Sevilla, D., Puerta, V. y Dávila, J. (2010). Influencia de los factores socioeconómicos en la deserción estudiantil de la carrera de Ciencias Sociales. Revista para el diálogo cientifico e intercultural, 6(1), 72-84. Recuperado de https:/ $/$ dialnet.unirioja.es/servlet/articulo?codigo $=6576527$

Vargas, H. y Huamán, I. (2010). Características de los intereses vocacionales de los alumnos del 2do año y 3er año de la escuela de ingeniería en informática y sistemas de la facultad de ciencias de la Universidad Nacional Jorge Basadre Grohmann de Tacna- Año académico 2009. Ciencia y Desarrollo 13(1), 68-71. doi: https://doi.org/1 $0.33326 / 26176033.2011 .13 .281$

Woyno, W. y Oñoro, R. (2005). Escala de preferencias Kuder Vocacional. México: Manual Moderno.

\section{INFORMACIÓN ADICIONAL}

Cómo citar: Alfaro-Barquero, A. y Chinchilla-Brenes, S. (2021). Prueba psicométrica de perfiles vocacionales de Administración de Tecnología de Información, Agronegocios y Arquitectura: construcción y validación. Revista Educación, 45(2). Recuperado de http://doi.org/10.15517/revedu.v45i1.43570 